\title{
TWIST MAPS, COVERINGS AND BROUWER'S TRANSLATION THEOREM
}

BY

\author{
H. E. WINKELNKEMPER ${ }^{1}$
}

\begin{abstract}
We apply the Brouwer Translation Theorem to a class of twist maps of the annulus (which contains $C^{1}$ area preserving maps) to show that, if $h$ belongs to this class, then a certain set $\mathscr{P}_{0}$ of periodic points of $h$ cannot be dense. The definition of $\mathscr{P}_{0}$ does not impose any a priori restrictions on the periods of the points of $\mathscr{P}_{0}$.
\end{abstract}

Introduction. Let $h: R^{2} \rightarrow R^{2}$ be an orientation-preserving, fixed point free, self-homeomorphism of the two-dimensional plane.

The weakest version of Brouwer's Translation Theorem (see e.g. [1] $)^{2}$ states: if $A \subset R^{2}$ is an arc-wise connected set such that $A \cap h(A)=\varnothing$, then $A \cap h^{n}(A)=$ $\varnothing$ for all $n \neq 0$. Here $h^{n}$ denotes the $n$-fold iterate of $h$.

In order to see whether this strong result on the behavior of all iterates of $h$ (i.e., a result of dynamics) has any relevance for the dynamics on other surfaces (especially compact surfaces, where all this is more interesting) it is natural to proceed as follows: Let $h: M \rightarrow M$ be an orientation-preserving self-homeomorphism of a connected, orientable two-manifold $M$ (with or without boundary $\partial M$ ) such that its fixed point set, Fix $h$, does not separate $M$; let $M_{0}=M-(\partial M \cup$ Fix $h)$ and $h_{0}=h \mid M_{0}$; then, since the universal cover $\tilde{M}_{0}$ of $M_{0}$ is homeomorphic to $R^{2}$ and any lifting to $\tilde{M}_{0}, \tilde{h}_{0}: \tilde{M}_{0} \rightarrow \tilde{M}_{0}$, of $h_{0}$ is orientation-preserving and fixed point free, we can apply Brouwer's Translation Theorem to $\tilde{h}_{0}$ and ask whether, in this way, we obtain any relations downstairs on the dynamics of $h_{0}: M_{0} \rightarrow M_{0}$, which is the same as that of the original homeomorphism $h: M \rightarrow M$.

We prove that the answer is yes, by using this idea to find a relation on a certain set $\mathscr{P}_{0}$ of periodic points of $h$, when $h: A \rightarrow A$ is a twist homeomorphism, which satisfies a mild purely homotopy-theoretical condition (see Definition 1.1, below). The relation is that $\mathscr{P}_{0}$ cannot be dense and so, in particular, if $h$ is also topologically transitive, $\mathscr{P}_{0}$ is nowhere dense.

If $h$ is at least $C^{1}$, then the same proof shows that $\cup H_{0}(P)$, the union taken over all hyperbolic $P \in \mathcal{P}_{0}$, is not dense either. Here $H_{0}(P)$ denotes a certain set of homoclinic points of $P$ (see Definition 1.3, below).

We thank the referee for very helpful comments.

Received by the editors December 15, 1979 and, in revised form, August 1, 1980.

1980 Mathematics Subject Classification. Primary 57N05; Secondary 58F11, 28D05.

Key words and phrases. Twist map, Brouwer Translation Theorem, transitive, periodic points, covering space.

${ }^{1}$ Partially supported by NSF Grant MCS77-02721.

2 Also S. A. Andrea, Trans. Amer. Math. Soc. 151 (1970), 481; Bull. Amer. Math. Soc. 71 (1965), 381. 
1. Statement of the Theorem. Let $A=\left\{(x, y) \in R^{2} \mid 0<a^{2} \leqslant x^{2}+y^{2}<b^{2}\right\}$ denote a planar annulus and $h: A \rightarrow A$ an orientation-preserving homeomorphism which maps each component of the boundary of $A$ onto itself. Let $\bar{A}$ denote the band $\bar{A}=\left\{(x, y) \in R^{2} \mid a \leqslant y \leqslant b\right\}$, which we regard as the universal cover of $A$ and $p: \bar{A} \rightarrow A$ the projection.

Recall that $h: A \rightarrow A$ is called a twist map if $h$ has a lifting $\bar{h}: \bar{A} \rightarrow \bar{A}$ which maps the points of the parallel lines $y=a, y=b$ in opposite directions.

The simplest examples of twist maps are obtained by integrating the flow shown in Figure 1.1.

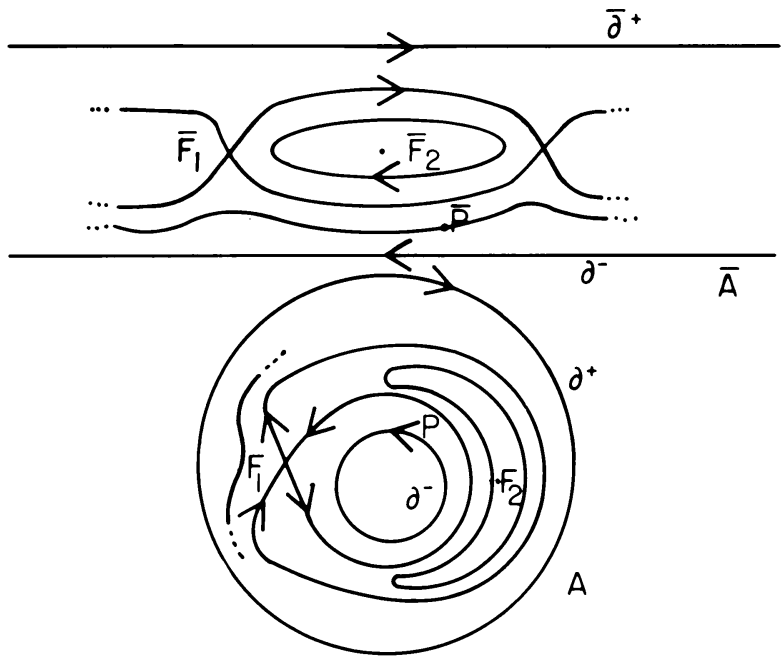

Figure 1.1

Notice that $h$ itself can have fixed points on the boundary of $A$ and that the set of twist maps is open in the space of all homeomorphisms of $A$, furnished with the $C^{0}$ topology.

Definition 1.1. Let $h: A \rightarrow A, \bar{h}: \bar{A} \rightarrow \bar{A}$ be a twist map and assume the fixed point set of $\bar{h}$, Fix $\bar{h}$, is not empty and does not separate $\bar{A}$; let $\overline{A_{0}}=\bar{A}-$ Fix $\bar{h}$, $\bar{h}_{0}=\bar{h} \mid \bar{A}_{0}$ and $\tilde{A}_{0}$ be the universal cover of $A_{0}$. We say $h: A \rightarrow A$ satisfies condition $\mathrm{L}$ if $\bar{h}_{0}: \bar{A}_{0} \rightarrow \bar{A}_{0}$ has a lifting $\tilde{h}_{0}: \tilde{A}_{0} \rightarrow \tilde{A}_{0}$ which sends every boundary component of $\partial \tilde{A}_{0}$ onto itself.

EXAMPLE. It is clear that any diffeomorphism obtained by integrating the flow of Figure 1.1 satisfies condition $L$.

We remark that condition $\mathrm{L}$ is a purely homotopy-theoretical condition: it holds if and only if the maps induced by $\bar{h}$ on $\Pi_{1}\left(\bar{A}_{0}, \bar{\partial}^{+}\right)$and $\Pi_{1}\left(\bar{A}_{0}, \bar{\partial}^{-}\right)$are the identity; here $\bar{\partial}^{+}, \bar{\partial}^{-}$denote the upper and lower boundary components of $\bar{A}_{0}$ and $\Pi_{1}(X, Y)$ denotes the homotopy classes of arcs in $X$ with endpoints in $Y$; the homotopies should keep endpoints in $Y$.

We now assume without loss of generality that $h, \bar{h}$ is a twist map such that $\bar{h}$ sends points of the lower boundary $\bar{\partial}^{-}$of $\bar{A}$ to the left. Let $T: \bar{A} \rightarrow \bar{A}$ be the generator of the covering transformation group which sends all points of $\bar{A}$ to the right. If $P \in A$ is a periodic point of $h$ of period $n$ and $\bar{P} \in \bar{A}$ lies above $P$, then there 
exists a unique integer $m=m(P)$ such that $T^{m}\left(\bar{h}^{n}(\bar{P})\right)=\bar{P}$ and $m$ does not depend on which point $\bar{P}$ lying over $P$ we chose.

Let $p: \bar{A} \rightarrow A$ be the covering projection and $h: A \rightarrow A, \bar{h}: \bar{A} \rightarrow \bar{A}$ a twist map (such that $\bar{h}$ sends points of $\bar{\partial}-$ to the left).

Definition 1.2. We say the periodic point $P$ of $h$ (of period $n$ ) belongs to the set $\mathscr{P}_{0}$ if in $A_{0}=A-p($ Fix $\bar{h})$ there exists an arc $\gamma$ from $P$ to $\partial^{-}$such that

(a) $m(P)>0$,

(b) $\gamma$ and $h^{n}(\gamma)$ are homotopic in $A_{0}$ by a homotopy, which keeps $P$ fixed and the endpoints of $\gamma$ and $h^{n}(\gamma)$ on $\partial^{-}$,

(c) $\gamma$ has a lifting, $\bar{\gamma}$, to $\bar{A}$, such that

$$
\bar{h}(\bar{\gamma}) \cap \bar{\gamma}=\varnothing .
$$

For example, in Figure 1.1 one can easily find a diffeomorphism such that $P$ is a periodic point $\in \mathscr{P}_{0}$ with $m(P)=1$.

If the boundary component $\partial^{-}$were a fixed point then (b) means that $P$ and $\partial^{-}$ lie in the same Nielsen fixed point class with respect to the map $h_{0}^{n}: A_{0} \rightarrow A_{0}$.

Recall that $h \leqslant A \rightarrow A$ is called topologically transitive if some point has a dense orbit with respect to $h$. It is an elementary fact that a nonempty, open, invariant set $\Omega$ (i.e. $h(\Omega) \subset \Omega$ ) of a transitive map is dense in $A$.

We can now state

THEOREM. Let $h: A \rightarrow A, \bar{h}: \bar{A} \rightarrow \bar{A}$ be a twist map which satisfies condition $\mathrm{L}$ and such that $\bar{h}$ sends points of $\bar{\partial}-$ to the left. Then there exists an open neighborhood $\Omega$ of $\partial^{+}$such that $\mathscr{P}_{0} \cap \Omega=\varnothing$. In particular, if $h$ is also transitive, then $\mathscr{P}_{0}$ is nowhere dense in $A$ (i.e., its complement contains an open dense subset of $A$ ).

Remark $1 . \Omega$ can be taken to be the subset of all $x \in A_{0} \subset A$ which can be joined to $\partial^{+}$by a free $\operatorname{arc} \alpha$, i.e., an $\operatorname{arc} \alpha$ such that $\alpha \cap h(\alpha)=\varnothing$.

REMARK 2. If $\mathscr{P}_{0}(N)=\left\{P \in \mathscr{P}_{0} \mid\right.$ period of $\left.P \leqslant N\right\}$ then for each $N$ the theorem is obvious with respect to $\mathscr{P}_{0}(N)$. Thus the point is that an $\Omega$ independent of $N$ exists.

Remark 3. The theorem is a purely topological theorem; no differentiability or measure-preserving assumptions of any kind are required for $h$.

Let $h$ now be at least $C^{1}$ and let $P$ be a hyperbolic periodic point (of period $n$ ) of $h$; then the sets

$$
W^{s}=W^{s}(P)=\left\{x \in A \mid \lim _{k \rightarrow+\infty} h^{n k}(x)=P\right\}
$$

and

$$
W^{u}=W^{u}(P)=\left\{x \in A \mid \lim _{k \rightarrow-\infty} h^{n k}(x)=p\right\}
$$

are called, respectively, the stable and unstable manifolds of $P$. It is well known that $W^{s}$ and $W^{u}$ are the images of $C^{1}$ injective immersions $R \rightarrow A$. A point of intersection of $W^{s}(P)$ and $W^{u}(P)$, other than $P$, is called a homoclinic point of $P$.

Let $H$ be a homoclinic point of $P$ (with respect to $h: A \rightarrow A$ ) and let $\beta^{s}\left(\beta^{u}\right)$ be the arcs, in $W^{s}\left(W^{u}\right)$, from $H$ to $P$. Assume $P \in \mathscr{P}_{0}$ and let $\gamma$ be as in Definition 1.2 . 
Definition 1.3. We say $H$ is inessential (we write $H \sim 0$ ) if the $\operatorname{arc} \gamma \beta^{u} \beta^{s} \gamma^{-1}$ has a lifting to $\bar{A}_{0}$ which is homotopic to 0 in $\pi_{1}\left(\bar{A}_{0}, \bar{\partial}^{-}\right)$keeping endpoints on $\bar{\partial}^{-}$.

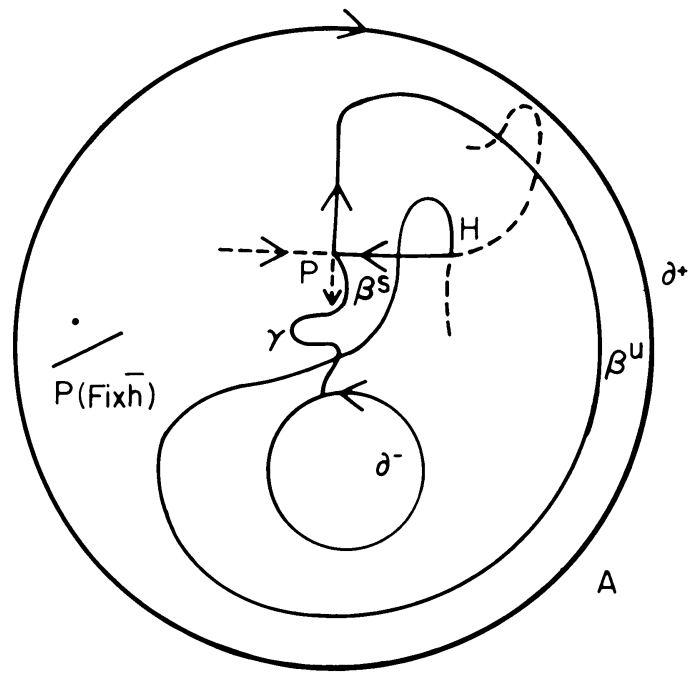

FIGURE 1.2

Define $H_{0}(P)$ as the set of inessential homoclinic points of $P$.

COROllary TO THE THEOREM. If $h$ is $C^{1}$ and satisfies the hypothesis of the Theorem, then there exists an open neighborhood $\Omega$ of $\partial^{+}$such that $\mathcal{H}_{0} \cap \Omega=\varnothing$; here $\mathcal{H}_{0}=\cup \mathcal{K}_{0}(P)$, the union taken over all hyperbolic $P \in \mathcal{P}_{0}$. (We can take $\Omega$ as in the theorem; see Remark 1.)

2. Proof of the Theorem. Since Fix $\bar{h} \neq \varnothing$, the boundary of $\tilde{A}_{0}, \partial \tilde{A}_{0}$, consists of an infinite number of components, each homeomorphic to the real line. We denote a component of $\partial \tilde{A}_{0}$ lying over $\partial^{+}$or $\partial^{-}$by $\tilde{\partial}^{+}$or $\tilde{\partial}^{-}$, and call them opposite boundaries if they lie above opposite boundaries of $A$. We know there exists a lifting $\tilde{h}_{0}: \tilde{A}_{0} \rightarrow \tilde{A}_{0}$ of $\overline{h_{0}}: \overline{A_{0}} \rightarrow \bar{A}_{0}$ which is orientation-preserving, fixed point free and takes each boundary component of $\partial \tilde{A}_{0}$ onto itself; therefore we can draw on each such component $\tilde{\partial}$ an arrow which indicates in which direction $\tilde{h_{0}} \mid \tilde{\partial}$ moves the points of $\tilde{\partial}$.

Assertion 1. If $\lambda$ is any arc in $\tilde{A}_{0}$ joining opposite boundaries $\tilde{\partial}^{+}, \tilde{\partial}^{-}$with endpoints $\tilde{Q}^{+}, \tilde{Q}^{-}$, then $\tilde{h}_{0}\left(\tilde{Q}^{+}\right)$and $\tilde{h}_{0}\left(\tilde{Q}^{-}\right)$lie in different (unbounded) components of $\tilde{A}_{0}-\lambda$; i.e., the arrows are as shown in Figure 2.1.

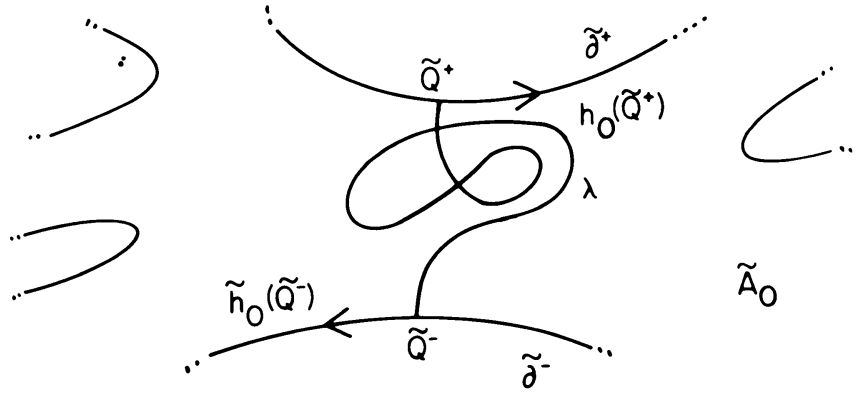

FIGURE 2.1 
Indeed, since $h$ is a twist map we can orient $\overline{A_{0}}$ in such a way that the direction in which $\bar{h}_{0}$ sends the boundaries $\bar{\partial}^{+}, \bar{\partial}^{-}$coincides with the induced orientations on them. Since $\tilde{A}_{0}$ covers $\overline{A_{0}}$ and $\tilde{h}_{0}$ is an orientation-preserving lifting, the same will hold for any two boundaries $\tilde{\partial}^{+}, \tilde{\partial}^{-}$lying over $\bar{\partial}^{+}, \bar{\partial}^{-}$.

Let $P \in \mathscr{P}_{0}$ via the arc $\gamma$ and assume without loss of generality ${ }^{3}$ that $m(P)=1$ (see Definition 1.2). If $P$ were sufficiently close to $\partial^{+}$, then there would exist an arc $\alpha \subset A_{0}$ from $P$ to $\partial^{+}$, and a lifting, $\bar{\alpha} \subset \overline{A_{0}}$, to the band $\bar{A}$ of $\alpha$ such that $\bar{h}_{0}(\bar{\alpha}) \cap \bar{\alpha}=\varnothing$ (Figure 2.2). This is clear because $\bar{h}_{0} \mid \bar{\partial}+$ is equivariant and fixed point free and $\partial^{+}$is compact.

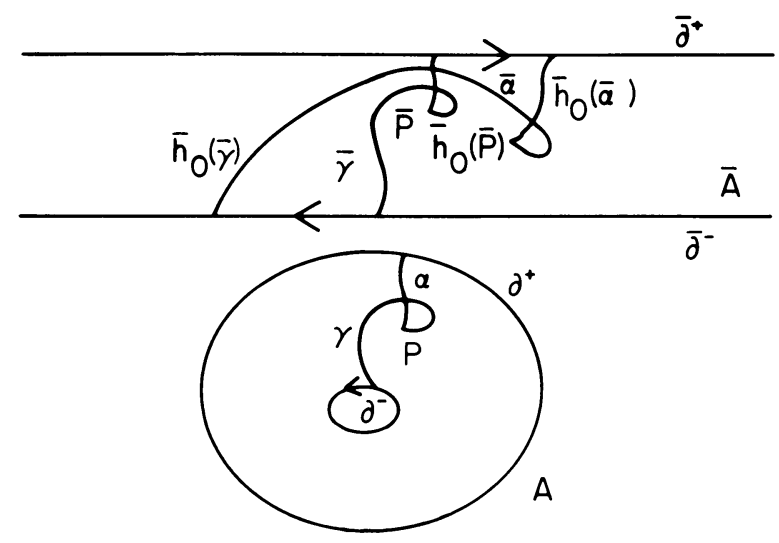

Figure 2.2

Assuming the existence of $\alpha$, we will arrive at a contradiction:

Let $Q^{-}$be the initial point of $\gamma$; we pick a boundary component $\tilde{\partial}_{0}$ of $\partial \tilde{A}_{0}$ and on it a point $\tilde{Q}_{0}^{-}$lying over $Q^{-}$. Lift the arc $\gamma$ to an arc $\tilde{\gamma}_{0} \subset \tilde{A}_{0}$ with initial point $\tilde{Q}_{0}^{-} \in \tilde{\partial}_{0}^{-}$; then the endpoint of $\tilde{\gamma}_{0}, \tilde{P}_{0}$, will lie over $P$ (Figure 2.3 ). Let $\tilde{Q}_{1}^{-}$be the next point (in the direction indicated) lying over $Q^{-}$and let $\tilde{P}_{1}$ be obtained by again lifting $\gamma$ to $\tilde{A}_{0}$, but now with initial point $\tilde{Q}_{1}^{-}$, to an $\operatorname{arc} \tilde{\gamma}_{1}$ with endpoint $\tilde{P}_{1}$, which again lies over $P$.

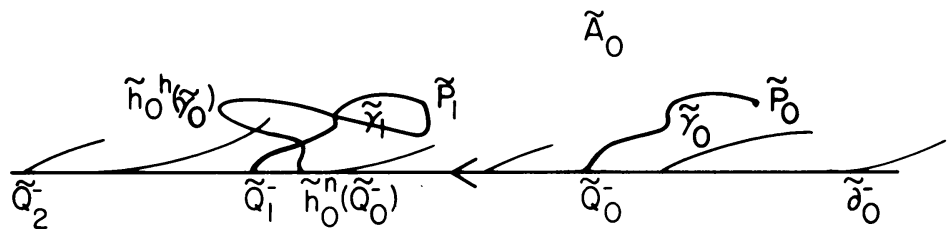

Figure 2.3

Similarly obtain $\tilde{Q}_{k}^{-}, \tilde{\gamma}_{k}, \tilde{P}_{k}$, also for negative $k$ in the obvious way.

ASSERTION 2. $\tilde{h}_{0}^{k n}\left(\tilde{P}_{0}\right)=\tilde{P}_{k}$ for all integers $k$.

Indeed, by the covering homotopy property, this is an immediate consequence of the facts that $P \in \mathscr{P}_{0}$ via $\gamma$ (Definition 1.2(b)) and $\tilde{h}_{0}$ maps $\tilde{\partial}_{0}$ onto itself (Figure 2.3).

\footnotetext{
${ }^{3}$ In the sense that the proofs for $m(P)>1$ are entirely similar.
} 
Remark. The point of Assertion 2 is: With respect to the induced riemannian metric of $\tilde{A}_{0}$, the iterate $\tilde{h}_{0}^{k n}\left(\tilde{P}_{0}\right)$ is, for all $k$, at a bounded distance from $\tilde{Q}_{k}^{-}$, namely bounded by the length of $\tilde{\gamma}_{k}$, i.e., the length of $\gamma$, which does not depend on $k$.

Let $\tilde{\partial}_{0}^{+}$be the boundary component of $\partial \tilde{A}_{0}$ determined by lifting the arc $\gamma \cup \alpha$ (Figure 2.2) to an $\operatorname{arc} \lambda=\tilde{\gamma}_{0} \cup \tilde{\alpha}_{0}$ (Figure 2.4) with initial point $\tilde{Q}_{0}^{-}$and endpoint $\tilde{Q}_{0}^{+} \in \tilde{\partial}_{0}^{+}$. By Assertion 1, the arrows are as shown in Figure 2.4 and $\lambda$ divides $\tilde{A}_{0}$ into two unbounded components.

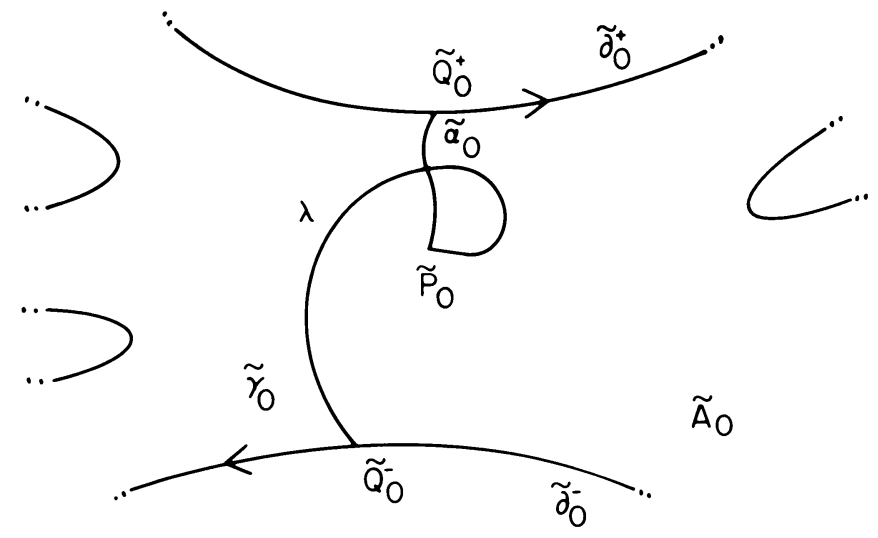

FIGURE 2.4

By Assertion 2 and the compactness of $\lambda$ and the bounded regions formed by $\lambda$, if $k>0$ is sufficiently large, the points $\tilde{h}_{0}^{k n}\left(\tilde{P}_{0}\right)=\tilde{P}_{k}$ and $\tilde{h}_{0}^{-k n}\left(\tilde{P}_{0}\right)=\tilde{P}_{-k}$ will lie in different, unbounded components of $\tilde{A}_{0}-\lambda, \tilde{P}_{k}$ lying in that component where $\tilde{h}_{0}^{k n}\left(\tilde{Q}_{0}^{-}\right)$lies. Moreover, since $\tilde{\alpha}_{0}$ and $\tilde{\gamma}_{0}$ are free under $\tilde{h}_{0}$, by the weak form of Brouwer's Translation Theorem [1, Satz 7, p. 13] they are still free under $\tilde{h}_{0}^{k n}$, where $k \neq 0$ is arbitrary.

We now have the following situation (Figure 2.5).

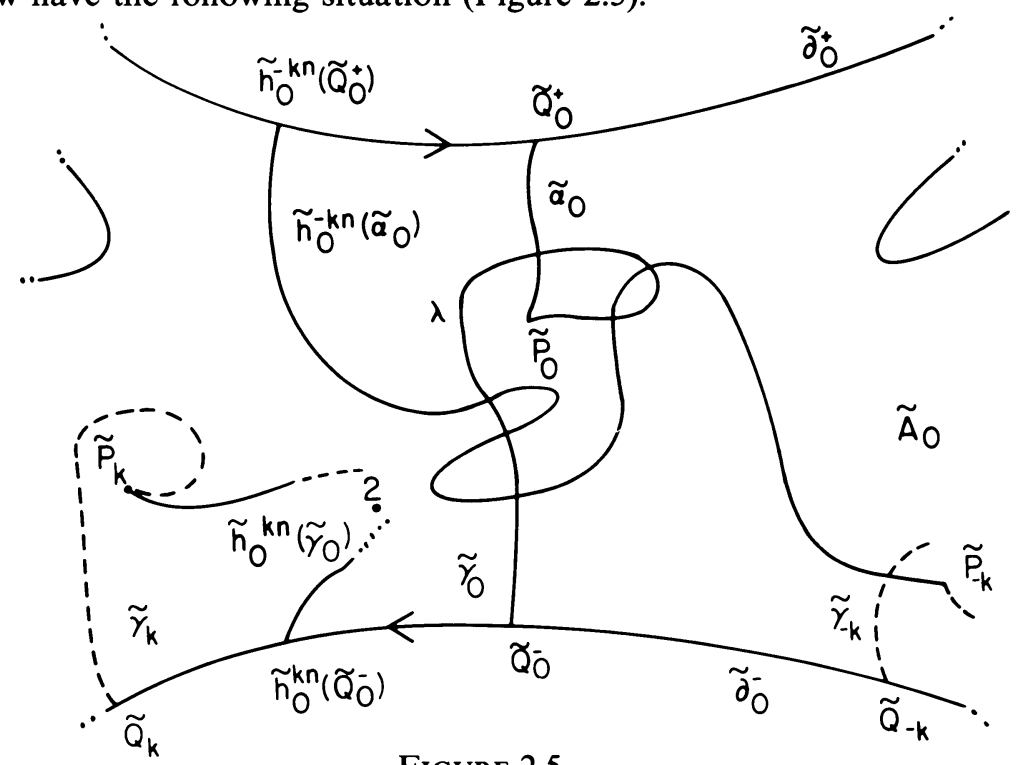

FIGURE 2.5 
If $k>0$ is sufficiently large, the arc ${\tilde{h_{0}}}^{-k n}\left(\tilde{\alpha}_{0}\right)$ has to intersect the dividing arc $\lambda=\tilde{\gamma}_{0} \cup \tilde{\alpha}_{0}$ an odd number of times, because by Assertions 1 and 2, its endpoints $\tilde{h}_{0}^{-k n}\left(\tilde{Q}_{0}^{+}\right)$and $\tilde{P}_{-k}$ lie in different unbounded components of $\tilde{A}_{0}-\lambda$. Since $\tilde{h}_{0}^{-k n}\left(\tilde{\alpha}_{0}\right)$ cannot intersect $\tilde{\alpha}_{0}$ it intersects $\tilde{\gamma}_{0}$ an odd number of times. Therefore the arc $\tilde{h}_{0}^{k n}\left(\tilde{\gamma}_{0}\right)$ has to intersect $\tilde{\alpha}_{0}$ an odd number of times, but since $\tilde{h}_{0}^{k n}\left(\tilde{\gamma}_{0}\right)$ cannot intersect $\tilde{\gamma}_{0}$, it intersects the whole dividing arc $\lambda$ an odd number of times, which is impossible, because its endpoints $\tilde{P}_{k}$ and $\tilde{h}_{0}^{k n}\left(\tilde{Q}_{0}^{-}\right)$lie in the same component of $\tilde{A}_{0}-\lambda$ (Figure 2.5).

We have shown $\mathscr{P}_{0}$ cannot intersect a small enough neighborhood of $\partial^{+}$and our theorem is proven.

Proof of the COROllary. Let $H \in H_{0}(P)$, and assume an arc $\alpha$ for $H$ exists. By iterating $H$, if necessary, we can suppose, without loss of generality, that the arc $\gamma \cup \beta^{s}$ is such that a lifting of it to $\overline{A_{0}}$ does not intersect its image under $\overline{h_{0}}$. Let $\tilde{H}_{0} \in \tilde{A}_{0}$ be the endpoint of the lifting, $\tilde{\gamma}_{0} \cup \tilde{\beta}_{0}^{s}$, of $\gamma \cup \beta_{0}^{s}$ to $\tilde{A}_{0}$ with initial point $\tilde{Q}_{0}^{-}$. The facts that $H \sim 0$ (see Definition 1.3) and that $\beta^{s}, \beta^{u}$ are mapped into themselves under $h^{n}, h^{-n}$ imply that, for large $k, \tilde{h}_{0}^{k n}\left(\tilde{H}_{0}\right)$ and $\tilde{h}_{0}^{-k n}\left(\tilde{H}_{0}\right)$ lie in different unbounded components of $\tilde{A}_{0}-\tilde{\gamma}_{0} \cup \tilde{\beta}_{0}^{s} \cup \tilde{\alpha}_{0}$ and the argument proceeds as before (Figure 2.6). Here $\tilde{P}_{0}^{\prime}$ denotes the endpoint of the lifting, $\tilde{\beta}_{0}^{u}$, to $\tilde{A}_{0}$ of $\beta^{u}$ with initial point $\tilde{H}_{0}$; since $H \sim 0, \tilde{P}_{0}^{\prime}=\tilde{P}_{k}$ for some $k$.

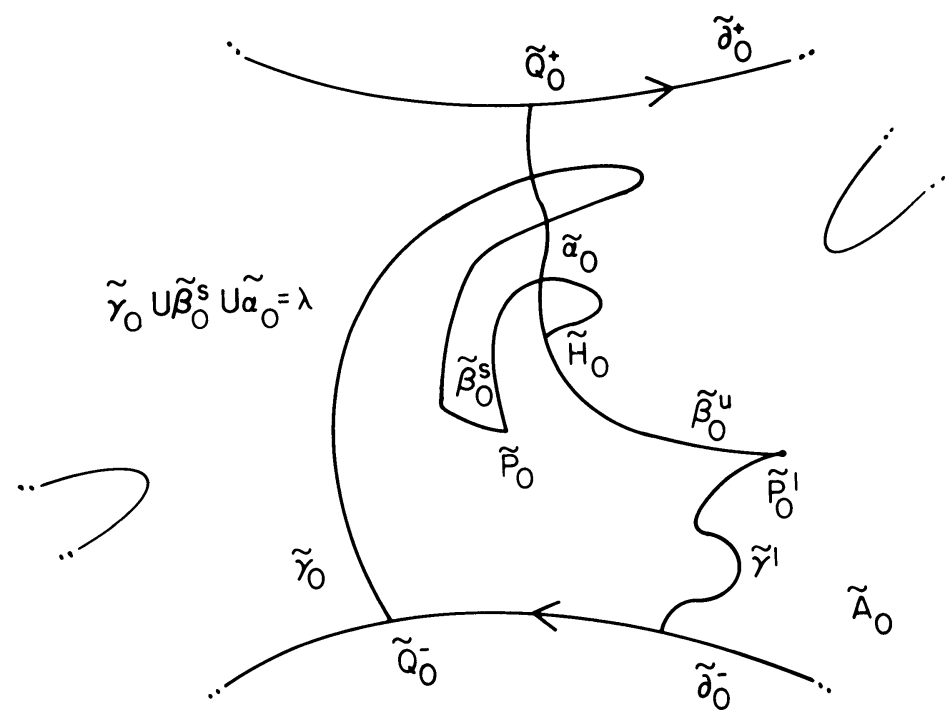

FIGURE 2.6

REMARK. In the proof of the corollary notice that in order to arrive at a contradiction to the existence of $\alpha$ it is enough that the lifting of $\gamma \beta^{u} \beta^{s} \gamma^{-1}$ to $\bar{A}_{0}$ of Definition 1.3 determines a boundary component $\tilde{\partial}_{0}^{\prime}$ of $\tilde{A}_{0}$ which lies in the right-hand side (unbounded) component of $\tilde{A}_{0}-\tilde{\gamma}_{0} \cup \tilde{\beta}_{0}^{s} \cup \tilde{\alpha}_{0}$ (see Figure 2.7). 


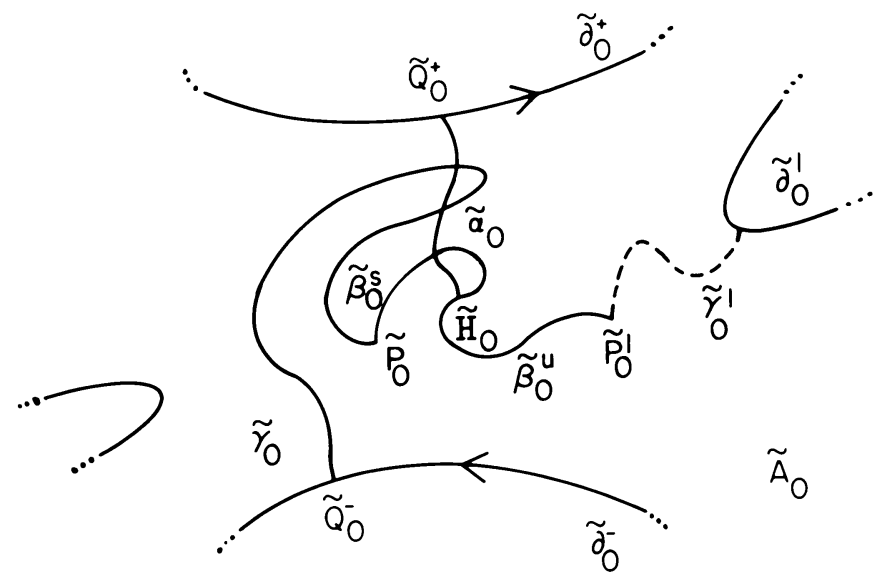

FIGURE 2.7

3. Remarks. (i) In the (not so interesting) case that the twist map $h: A \rightarrow A$ is fixed point free, our theorem takes the form:

$$
\mathscr{P} \cap S^{+} \cap S^{-}=\varnothing
$$

where $\mathscr{P}$ denotes the set of all periodic points of $h$ and $S^{+}, S^{-}$are the projections into $A$ of the sets $\bar{S}^{+}, \bar{S}^{-} \subset \bar{A}$ defined as follows: $\bar{X} \in \bar{A}$ lies in $\bar{S}^{+}, \bar{S}^{-}$if there exists an arc $\bar{\alpha}^{+}\left(\bar{\alpha}^{-}\right)$from $\bar{X}$ to $\bar{\partial}^{+}(\bar{\partial}-)$ such that $\bar{h}\left(\bar{\alpha}^{+}\right) \cap \bar{\alpha}^{+}=\varnothing\left(\bar{h}\left(\bar{\alpha}^{-}\right) \cap \bar{\alpha}^{-}\right.$ $=\varnothing$ ).

In the case that $h$ is fixed point free, twist maps seem to have been first related to Brouwer's Translation Theorem by Kerèkjartò, The plane translation theorem of Brouwer and the last geometric theorem of Poincaré, Acta Sci. Math. Szeged 4 (1928), 86-102.

(ii) The following is a sufficient metric condition for $h$ to satisfy condition L: Assume $h: A \rightarrow A$ has only a finite number of fixed points in $A$ and $|h(z)-z| \leqslant \alpha$ for all $z \in A$ for some $\alpha$ such that $0<\alpha<\Pi a$; then if $h$ does not satisfy condition $\mathrm{L}$, there exists a fixed point $F$ such that either:

(a) in an $\alpha$-neighborhood of $F$ there exists a point $z$ such that the angle between the segments $\overline{z F}$ and $\overline{h(z) F}$ is $\Pi$, or

(b) a closed neighborhood of $F$ intersects the boundary of $A$ or a closed $2 \alpha$-neighborhood of $F$ contains a fixed point different from $F$.

To see this, it is enough to show that in $A_{0}$ there is a unique shortest arc joining any $z \in A_{0}$ to $h_{0}(z)$, because then a good $\tilde{h}_{0}$ will be obtained by lifting $h_{0}$ along these arcs. The negation of the conclusions (a), (b) above immediately shows that outside of an $\alpha$-neighborhood of the inner boundary of $A$, we can join $z$ to $h_{0}(z)$, in $A_{0}$, by the straight line segment $\overline{z h_{0}(z)}$. In an $\alpha$-neighborhood of the inner boundary, since $\alpha<\Pi a$, there also is a unique shortest arc from $z$ to $h_{0}(z)$ (Figure 3.1). 


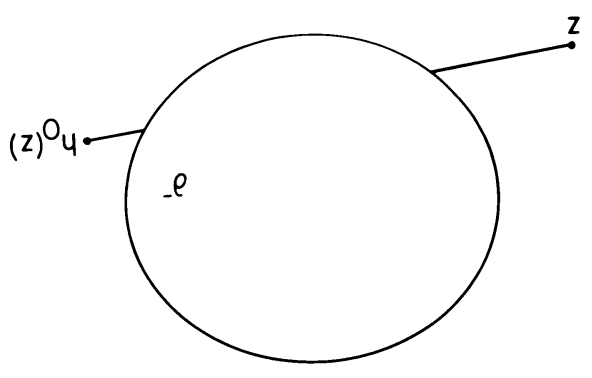

Figure 3.1

(iii) Let $h: A \rightarrow A$ be a $C^{1}$ twist diffeomorphism obtained by integrating the flow of Figure 1.1 such that the fixed points $F_{1}, F_{2}$ are transversal, i.e., in $A \times A$ the graph of $h$ intersects the diagonal transversely at $F_{1}$ and $F_{2}$. We already know $h$ satisfies condition $L$; however, by making $h$ coincide with a small rotation near the elliptic fixed point $F_{2}$ and taking time $t$ small enough, condition $\mathrm{L}$ will be satisfied because of the metric condition of (ii) above. It follows that any $C^{1} h^{\prime}: A \rightarrow A$ sufficiently $C^{1}$ close to $h$ will also satisfy condition $\mathrm{L}$, since its corresponding fixed points $F_{1}^{\prime}, F_{2}^{\prime}$, will, due to transversality, be near $F_{1}$ and $F_{2}$. Hence, at least in this case, condition $\mathrm{L}$ is stable under $C^{1}$ perturbations.

\section{REFERENCES}

1. E. Sperner, Über die fixpunkt freien Abbildungen der Ebene, Abh. Math. Sem. Univ. Hamburg 10 (1934), 1-47.

Department of Mathematics, University of Maryland, College Park, Maryland 20742 